\title{
RESEARCH PAPER \\ Gambling addiction in China: a survey of Chinese psychiatrists
}

\author{
Xiuqin Huang ${ }^{1}$ MD, Du Shijun ${ }^{2}$ MD and Sanju George ${ }^{3}$ MRCPsych
}

${ }^{1}$ Associate Professor of Psychiatry, Department of Psychological Medicine, General Hospital of Beijing Military Area Command Congsishitiao, Dongcheng District, Beijing, China ${ }^{2}$ Associate Professor of Psychiatry, Department of Addiction Medicine, General Hospital of Beijing Military Region No. 5 , Nanmencang, Dongsishitiao, Dongcheng District, Beijing, China

${ }^{3}$ Consultant and Senior Research Fellow in Addiction Psychiatry, The Bridge Clinic, Birmingham and Solihull Mental Health NHS Foundation Trust, Birmingham, UK email sanjugeorg sanju.george@bsmhft. nhs.uk

\begin{abstract}
We studied Chinese psychiatrists' understanding of gambling addiction, as well as their experiences of and confidence in assessing and treating these patients. To this end, we carried out a postal questionnaire survey of 110 psychiatrists working in China. A majority had seen people with gambling addiction in their practice but only 1 of the 110 psychiatrists had ever received any training in the management of the condition. A large majority of psychiatrists indicated that gambling addiction was an important public health problem and $71.8 \%$ said they would like to be more involved in its management. Much more needs to be done to improve the identification and treatment of gambling addiction in China.
\end{abstract}

Gambling as a recreational pursuit is common in most cultures. For most of those who engage in gambling it is merely a leisure activity and never becomes problematic. However, for a minority $-1 \%$ of the general population in most Western countries (Shaffer et al, 1999) - it develops into gambling addiction or pathological gambling. According to ICD-10 (World Health Organization, 1992), the essential diagnostic feature of pathological gambling is "persistently repeated gambling, which continues and often increases despite adverse consequences such as impoverishment, impaired family relationships and disruption of personal life'.

Gambling in China possibly dates back to the Xia dynasty (2000 to 1500 BC) (Wu Ping-an, 2006). The prevalence of gambling addiction in China is much higher than in any Western country, at $2.5-4 \%$ of the adult population. This is despite most forms of gambling being banned in mainland China, with the exception of state-run lotteries. These lotteries were first introduced in 1987 and continue to generate substantial revenue, which the government uses to fund public welfare activities. However, illegal gambling is very popular in mainland China and it is estimated that the size of the illegal gambling industry is at least 10 times that of legal gambling (lotteries). Given all this, it is surprising that there is very little treatment provision for gambling addiction in China. Indeed, gambling addiction does not have a place within mainstream Chinese psychiatry or even Chinese addiction psychiatry.

In this context, we carried out a survey of 110 psychiatrists in China. We sought to explore their 'exposure' to patients with gambling addiction, their ability to manage them, their understanding of gambling addiction, their perceived role, their confidence in getting involved and their views on commissioning services for gamblers. To our knowledge, this is the first survey of its kind in China.

\section{Method}

A brief questionnaire was devised by the authors. It consisted of some demographic questions (age, gender, number of years working as a psychiatrist, etc.), some questions with yes/no answers, some statements with the option of choosing responses from 'strongly agree' to 'strongly disagree', and two open-ended questions. A copy of the questionnaire is available from the corresponding author upon request.

Eighty of these questionnaires were sent to psychiatrists at two psychiatric hospitals in Beijing, and 25 were sent to psychiatrists in a psychiatric hospital in the Guangdong province; another 15 were sent to psychiatrists who were encountered by one of the authors at an academic conference. Completed questionnaires were returned by 110 of these 120 psychiatrists.

\section{Results}

The age distribution of the sample was as follows: $31-40$ years $(35 ; 31.8 \%)$; $41-50$ years $(60 ; 54.5 \%)$; $51-60(13 ; 11.8 \%)$; and aged 60 and above $(2 ; 1.8 \%)$. Sixty per cent $(66)$ of the sample were men and $40 \%$ (44) were women. Table 1 summarises their understanding of the problem of gambling addiction.

\section{Psychiatrists' 'exposure' to patients with gambling addiction}

Of the 110 psychiatrists, $61(55.4 \%)$ said they had seen patients with gambling addiction in their practice. The number of such patients seen varied: 41 psychiatrists had seen between 1 and $5 ; 15$ had seen between 6 and 10; and 5 had seen more than 10 gamblers. Equally important was that $50(45.4 \%)$ psychiatrists also said they had seen patients who did not meet the threshold for diagnosis but nevertheless had a gambling problem. Forty $(36.4 \%)$ of the surveyed psychiatrists said they had seen patients affected by the gambling of a third party.

Psychiatrists were also asked if they had had any training in the management of gambling addiction: only one had. 
Table 1

Psychiatrists' understanding of gambling addiction

\begin{tabular}{|c|c|c|c|c|c|}
\hline & $\begin{array}{l}\text { Strongly } \\
\text { agree }\end{array}$ & Agree & $\begin{array}{l}\text { Neither agree } \\
\text { nor disagree }\end{array}$ & Disagree & $\begin{array}{l}\text { Strongly } \\
\text { disagree }\end{array}$ \\
\hline Gambling is an important public health problem & $57(51.8 \%)$ & $41(37.3 \%)$ & $9(8.2 \%)$ & $3(2.7 \%)$ & 0 \\
\hline People with gambling addiction have significant psychiatric comorbidity & $22(20.0 \%)$ & $53(48.2 \%)$ & $27(24.5 \%)$ & $7(6.4 \%)$ & 0 \\
\hline Undetected comorbid gambling problems can adversely affect mental health & $21(19.1 \%)$ & $78(70.9 \%)$ & $9(8.2 \%)$ & $1(0.9 \%)$ & 0 \\
\hline Gambling addiction can negatively impact on family members & $62(56.4 \%)$ & $46(41.8 \%)$ & $1(0.9 \%)$ & 0 & $1(0.9 \%)$ \\
\hline
\end{tabular}

\section{Psychiatrists' roles in the management of gambling addiction}

In response to the question 'Would you like more involvement in the management of gambling?', 79 psychiatrists $(71.8 \%)$ said they would like to get involved.

When asked about the feasibility of "psychiatrists getting involved in the management of gambling addicts', 22.0\% strongly agreed, 60.9\% agreed, $10.9 \%$ neither agreed nor disagreed, $5.4 \%$ disagreed and $0.9 \%$ strongly disagreed.

Responses to the question 'What would encourage you to get more involved in the management of gamblers?' were: 'more knowledge' (77.8\%), 'more training' (7.3\%) and 'more resources' (7.3\%); some opted for more than one response.

\section{Discussion}

Before discussing some of our key findings and their implications, we acknowledge some limitations of our survey, specifically a relatively small sample size $(n=110)$, with its possible response bias. Nevertheless, we believe this survey provides useful information that could inform future plans for engagement of psychiatrists and the implementation of relevant training programmes for psychiatrists. Our findings could also serve as a useful baseline measure of psychiatrists' awareness of and attitudes towards gambling addiction. Yet another shortcoming, potentially limiting the generalisability of our findings, is that this survey was mostly limited to two geographical regions of China (61 of the respondents were working in Beijing and 23 were in Guanzhou). However, in our view, there is nothing to suggest that these Chinese psychiatrists are not representative of the profession across the country.

It was interesting to note that the majority of the psychiatrists $(55.4 \%)$ had seen patients with gambling addiction in their day-to-day practice, $45.4 \%$ had seen patients with gambling problems who did not meet the diagnostic criteria and $36.4 \%$ had seen patients affected by the gambling of a third party. This confirms the view that people with gambling problems do present to psychiatrists in China. However, we cannot comment on these patients' reasons for presentation, as this information was not captured in this survey. Despite this 'exposure' of psychiatrists to patients with gambling problems, it was shocking to find that only 1 of the 110 psychiatrists had ever received any training in the management of gambling addiction. This reveals a huge gap between need and provision that requires urgent attention.

Notwithstanding this lack of training, it was encouraging to note that most of those who were surveyed acknowledged gambling addiction as an addictive disorder $(82.7 \%)$, as an important public health disorder $(89.1 \%)$ and as a disorder with important negative consequences. An acknowledgement that gambling addiction is a problem warranting attention is a necessary minimum requirement to incentivise psychiatrists to participate in future teaching programmes. Equally encouraging was the proportion of psychiatrists who said they would like to receive further training in the management of gambling disorders in primary care $(71.8 \%)$. Furthermore, $83.0 \%$ of respondents also felt that it would be feasible for them to be involved in the management of gambling addiction. This presents a great opportunity to introduce programmes aimed at increasing the knowledge of professionals in this neglected area of addiction psychiatry.

\section{Conclusion}

We end with a call for more to be done in mainland China to help people with gambling addiction; Chinese psychiatry and its practitioners have an important role to play in driving this agenda forward. Increasing the knowledge of gambling addiction among Chinese psychiatrists through training and teaching may be a good starting point, and here international collaborations will be beneficial.

\section{References}

Shaffer, H. J., Hall, M. N. \& Vander Bilt, J. (1999) Estimating the prevalence of disordered gambling behavior in the US and Canada: a research synthesis. American Journal of Public Health, 89, 1369-1376

World Health Organization (1992) The ICD-10 Classification of Mental and Behavioural Disorders. Clinical Descriptions and Diagnostic Guidelines. WHO.

Wu Ping-an (2006) Study of Gambling Crime. Thesis, Xiangtan University. 